\title{
Definindo Orientações de Usabilidade para o Desenvolvimento de Objetos de Aprendizagem para TV Digital
}

\author{
Fábio de Jesus Lima Gomes \\ Pós-Graduação em Informática na Educação (PGIE) \\ Universidade Federal do Rio Grande do Sul (UFRGS) \\ fjlgomes@inf.ufrgs.br \\ José Valdeni de Lima \\ Instituto de Informática (II) \\ Universidade Federal do Rio Grande do Sul (UFRGS) \\ valdeni@inf.ufrgs.br \\ Rosane Aragón de Nevado \\ Faculdade de Educação (FACED) \\ Universidade Federal do Rio Grande do Sul (UFRGS) \\ rosane@edu.ufrgs.br
}

\begin{abstract}
RESUMO
O objetivo desta pesquisa é produzir orientações de usabilidade para ajudar no desenvolvimento de objetos de aprendizagem fáceis de uso que serão utilizados na TV Digital (TVD). Para a definição das orientações, os seguintes passos estão sendo e serão realizados: (a) identificação de problemas de interface de aplicações para TVD; (b) projeto e desenvolvimento de objetos de aprendizagem (protótipos) para TVD; (c) avaliação heurística dos protótipos desenvolvidos; (d) testes de usabilidade para os protótipos desenvolvidos. As orientações serão baseadas nos resultados dos testes de usabilidade realizados em laboratório.
\end{abstract}

Palavras-chave: TV Digital. Usabilidade. Objetos de Aprendizagem. T-learning.

\section{Defining Usability Guidelines to Develop Digital TV Learning Objects}

\begin{abstract}
The aim of this research is to produce usability guidelines that support the development of easy to use Digital TV (DTV) learning objects. To define the guidelines the following steps are being and will be conducted: (a) identification of recurring DTV interface design problems; (b) design and development of DTV learning objects (prototypes); (c) heuristic evaluation of the developed DTV prototypes; (d) usability tests of the developed DTV prototypes. The guidelines are based upon empirical lab-based usability test results for DTV prototypes.
\end{abstract}

Keywords: Digital TV. Usability. Learning Objects. T-learning.

\section{INTRODUÇÃO}

Baas et al (2001) citam alguns problemas relacionados ao e-learning baseado na web: (a) usuários se "perdem" no labirinto de links na Internet; (b) manter um curso baseado na web significa muito overhead; (c) estar on-line custa dinheiro; (d) velocidade de conexão com a Internet é limitada. 
Peraton et al (2000) complementam que o acesso a computadores é visto como o principal problema na área das novas tecnologias de informação e comunicação. Lee et al (2002) declaram que mais de $50 \%$ da população mundial não tem acesso à Internet.

No Brasil, o cenário é o mesmo, pois apesar da popularidade da Internet, o Brasil ainda não é um país on-line. Segundo o CGIBr (2006), no segundo trimestre de 2006, apenas $15 \%$ da população brasileira tinha acesso à internet via um computador doméstico. Por outro lado, o Ministério das Comunicações (2003) diz que cerca de 90\% dos domicílios brasileiros possuem receptores de TV. É nesse contexto que surge no Brasil a TVD, com todas as adversidades e incertezas de uma nova mídia.

O decreto (Brasil, 2006) que dispõe sobre a implantação da TVD no Brasil deixou claro que o uso da TVD para finalidades educacionais será um dos seus principais objetivos. Embora a TV seja usada há vários anos para finalidades educacionais no Brasil (por exemplo, TV Escola e Telecurso 2000), a TVD oferece um nível de interatividade, onde o aluno passa de um papel passivo de mero telespectador para um papel ativo na interação com o conteúdo transmitido pela TV.

Esta mudança de papel pode contribuir com o processo de aprendizagem, pois, de acordo com Piaget (1996), o conhecimento é construído através da interação do sujeito com o objeto fruto de seu interesse. Dessa forma, surge a possibilidade da utilização de objetos de aprendizagem na TVD.

Embora existam orientações sobre o desenvolvimento de conteúdo para TVD, não foi encontrado na literatura, algo semelhante relacionado à construção de objetos de aprendizagem para TVD. Tal ausência resultou no início desta pesquisa.

A próxima seção apresenta conceitos básicos sobre a TVD e uma breve descrição de sua atual situação no Brasil. A seção 3 conceitua $t$-learning e apresenta vantagens e desvantagens do uso da TV para a educação. A seção 4 apresenta conceitos de objetos de aprendizagem. Na seção 5, tem-se uma breve explicação sobre usabilidade e em particular, trata-se sobre usabilidade na TVD. Na seção 6, uma descrição do que se propõe neste artigo é mostrada. Por último, a seção 7 traz as conclusões.

\section{TV DIGITAL}

Segundo Parker (1999), a TVD é algo mais que imagens mais limpas e melhor qualidade de áudio; é uma eventual convergência de televisão, telefonia, Internet e computador pessoal em uma simples caixa, com a promessa de acesso extraordinário a todos os tipos de informação e comunicação interativa inimagináveis.

TVD se refere à transmissão de sinais em uma forma digital. A transmissão digital permite mesclar conteúdos de áudio e vídeo com dados binários. Assim, é possível entregar aplicações de software em um aparelho digital de TV ou em um settop box. O set-top box é responsável em receber o sinal digital usando um sintonizador digital e então convertê-lo para um formato analógico para ser visto em um aparelho analógico de TV.

Segundo Aarreniemi-Jokipelto (2006), a diferença-chave entre a TV analógica e a TVD é o nível de interatividade que a transmissão digital permite.

\subsection{TV Digital no Brasil}

O decreto $n^{\circ} .5 .820$, de 29 de junho de 2006 (Brasil, 2006), que dispõe sobre a implantação do Sistema Brasileiro de Televisão Digital Terrestre (SBTVD-T) na plataforma de transmissão e retransmissão de sinais de radiodifusão de sons e imagens, além de nortear a transição do sistema analógico para o digital, deixa claro que esse avanço tecnológico não se restringirá a uma simples troca de equipamentos para atender 
a interesses mercadológicos ou econômicos. O decreto demonstra interesse com o desenvolvimento da indústria nacional e com a inclusão digital (e social) por intermédio da TV, que tende a se tornar uma ferramenta com finalidades sociais, com a criação de canais destinados ao poder executivo, cidadania, educação e cultura.

O sucesso da TVD no Brasil pode estar diretamente relacionado ao sucesso da inclusão digital. Além de outros serviços, esta inclusão digital pode ser alcançada através da educação pela TV, que é chamada de t-learning.

\section{T-LEARNING}

Segundo Bates (2003), t-learning é o acesso a materiais de aprendizagem ricos em vídeo, através de uma TV ou de um dispositivo mais parecido com a TV do que um PC. T-learning é um subconjunto do e-learning, mas que pode significativamente melhorar a aprendizagem em uma forma que o e-learning baseado na Internet atualmente não faz.

Aarreniemi-Jokipelto (2006) destaca algumas razões para o uso da TVD para finalidades educacionais: (a) Acessibilidade; (b) Serviços interativos; (c) Independência de tempo e lugar; (d) Baixo limiar para uso inicial; (e) Aprendizagem sob demanda.

Aarreniemi-Jokipelto (2006) complementa que a TVD é de grande importância quando comparada à transmissão analógica, devido às suas características de feedback e comunicação bi-direcional. Além disso, a TVD suporta o e-learning e permite a aprendizagem quando a Internet não está disponível.

Lytras et al (2002) referindo especialmente ao t-learning, argumentaram que a alta penetração e aceitação da TV já tem estabelecido um mercado em potencial para a aceitação da TVD. Num país de dimensões continentais como o Brasil, com uma população bastante familiarizada com a TV, o veículo tem tudo para tornar-se um grande aliado nas estratégias de superação dos graves problemas educacionais, contribuindo de maneira significativa para a expansão do acesso ao sistema de ensino e sua democratização.

Damásio (2003) declara que a TVD tem o potencial para fornecer motivação e dedicação e é uma mídia eficaz para promover a aprendizagem na escola, em casa ou em qualquer outro lugar. Aproveitando este potencial, surge a possibilidade da utilização de objetos de aprendizagem na TVD.

\section{OBJETOS DE APRENDIZAGEM}

De acordo com a terminologia adotada pelo Learning Technology Standards Committee (LTSC) do Institute of Electrical and Electronics Engineers (IEEE) (IEEE, 2005), objeto de aprendizagem é definido como uma entidade, digital ou não digital, que pode ser usada e reutilizada ou referenciada durante um processo de suporte tecnológico ao ensino e aprendizagem.

Wiley (2001) define objetos de aprendizagem como elementos de um novo tipo de instrução baseada em computador construído sobre um novo paradigma da ciência da computação e que possam ser reutilizados para o suporte ao ensino.

Menezes et al (2006) acrescentam que quando o ambiente do objeto de aprendizagem é digital, pode-se agregar ao objeto o registro das operações realizadas pelo sujeito, permitindo assim que o professor possa conhecer as suas atividades, ainda que não esteja presente. A partir desses dados, o professor terá a possibilidade de acompanhar o desenvolvimento dos processos cognitivos presentes na interação do sujeito com o objeto de aprendizagem.

No entanto, Costabile et al (2005) afirmam que aplicações para e-learning, tais como, objetos de aprendizagem, devem ser fáceis de serem usados. Caso contrário, o 
tempo do estudante será desperdiçado com a aplicação, ao invés de ser aproveitado para aprendizagem. Costabile et al (2005) complementam que pessoas se recusam a usar uma interface de um sistema que seja rígida, lenta e desagradável e acabam interrompendo o curso. A facilidade de uso está relacionada à usabilidade.

\section{USABILIDADE}

A ISO (International Organization for Standardization) se refere à usabilidade como a eficácia, eficiência e satisfação com que usuários específicos podem atingir objetivos específicos em um ambiente particular (ISO, 1998). Eficácia significa que o usuário é capaz de realizar a tarefa pretendida, eficiência se refere ao tempo gasto na realização da tarefa e satisfação define o quanto o sistema é aceitável pelos usuários (Nielsen, 1993).

$\mathrm{Na}$ engenharia da usabilidade, a avaliação heurística é um método para encontrar problemas de usabilidade em um projeto de interface de usuário, a fim de que tais problemas possam ser tratados como parte de um processo iterativo de projeto. A avaliação heurística envolve um pequeno número de avaliadores que examinam a interface e julgam sua conformidade com princípios de usabilidade reconhecidos, chamados de heurísticas (Nielsen, 1994).

Outra parte importante na engenharia da usabilidade são os testes de usabilidade e consistem em uma técnica altamente utilizada para estimar o desempenho e a satisfação subjetiva do usuário com produtos e/ou serviços. Tais testes são sempre realizados para testar o produto e/ou serviço, não o usuário. Eles permitem dizer se usuários podem usar um produto e/ou serviço e como eles gostariam de usá-lo (Nielsen, 1993).

\subsection{Usabilidade na TV digital}

Um dos principais problemas encontrados na TVD é a complexa navegação de informação pelos telespectadores através do controle remoto, que tende a ser grande, consistindo de muitos botões e não tão fácil para usar (Nelson, 1999). Tal navegação pode causar frustração e irritação do usuário (Berglund et al, 2005). A facilidade de uso foi identificada como um dos fatores mais importantes para o sucesso da TVD (Choi et al, 2003; Freeman \& Lessiter, 2003).

Existem poucas orientações de usabilidade para TVD, mas nenhuma especificamente para o uso da TVD relacionado à construção de objetos de aprendizagem. As orientações existentes no projeto de serviços para TVD são as seguintes:

- $\quad$ Orientações para modelagem e produção de conteúdo para educação a distância (Aarreniemi-Jokipelto, 2006);

- $\quad$ Orientações de projeto de serviços fáceis de uso (Ahoren et al, 2006);

- $\quad$ Guia de estilo (BBC, 2002);

- $\quad$ Orientações para apresentação de serviços de notícias (Brecht \& Kunert, 2005);

- Guia de estilo para o projeto de serviços de para telespectadores idosos (Carmichael, 1999);

- Orientações de projeto de navegação para aplicações (Kunert \& Krömker, 2007);

- $\quad$ Guia para produtores de serviços (Rinnetmäki, 2004);

- Orientações de projeto de sistemas de tecnologia da comunicação e informação com acessibilidade (RNIB, 2005); 
T-learning também possui requisitos de usabilidade. No entanto, ainda não existe uma metodologia de avaliação consolidada para sistemas de e-learning (Costabile et al, 2005), nem para t-learning (Aarreniemi-Jokipelto, 2006).

\section{MATERIAL E MÉTODOS}

Para identificar problemas de usabilidade específicos para TVD, uma revisão de literatura foi realizada. Como resultado, vários problemas foram encontrados e no desenvolvimento dos objetos de aprendizagem (protótipos), tais problemas foram solucionados. Três protótipos serão utilizados: simulador de circuitos digitais, quiz e gangorra interativa (ver figuras 1, 2 e 3). Como a finalidade da pesquisa é definir orientações de usabilidade, apenas a usabilidade dos protótipos será avaliada, portanto, os protótipos foram implementados de forma que o usuário possa utilizar todos os recursos disponíveis através do dispositivo de interação com a TV, no caso, o controle remoto. Além disso, também serão avaliadas questões sobre a interface dos protótipos.

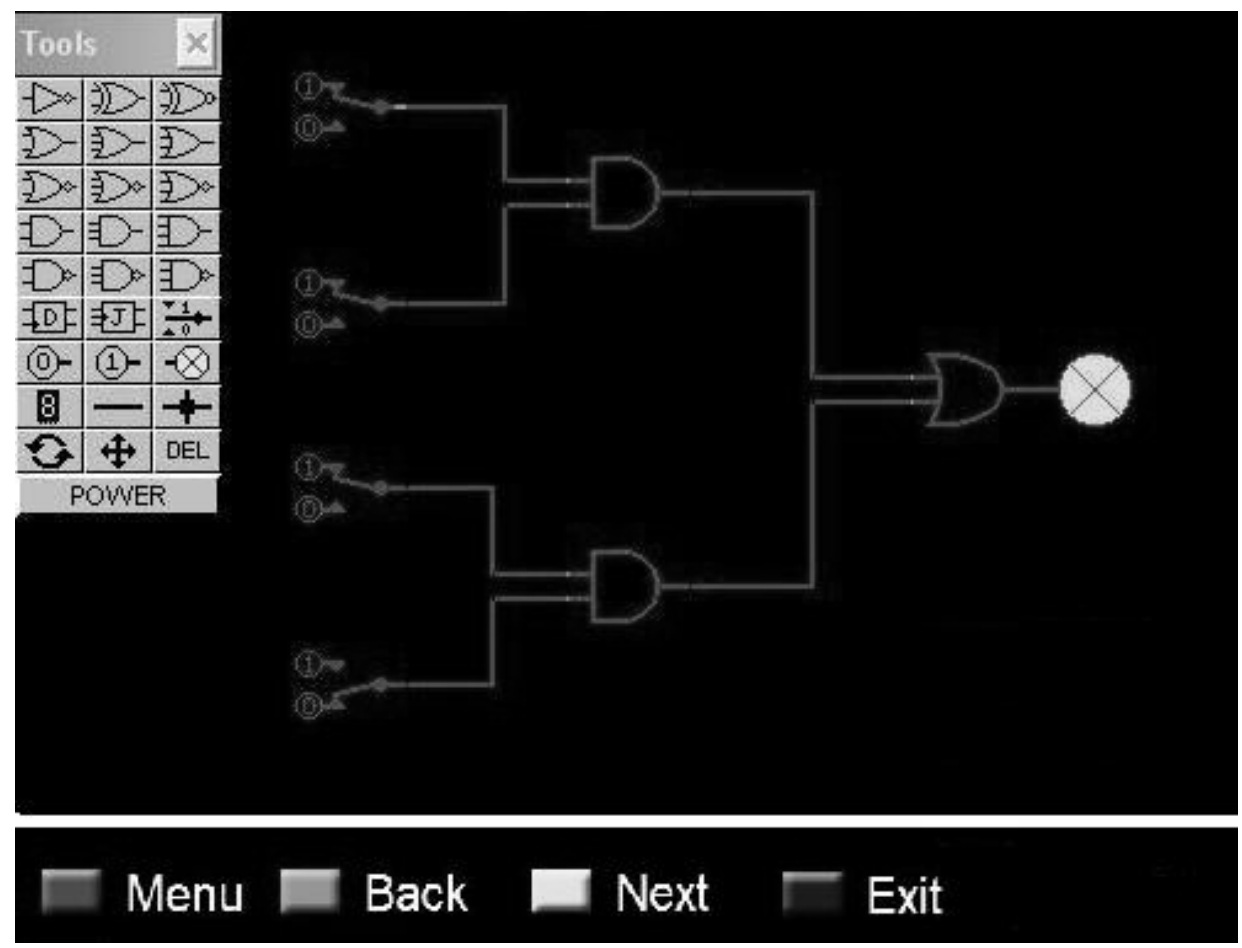

Figura 1. Tela do simulador de circuitos digitais.

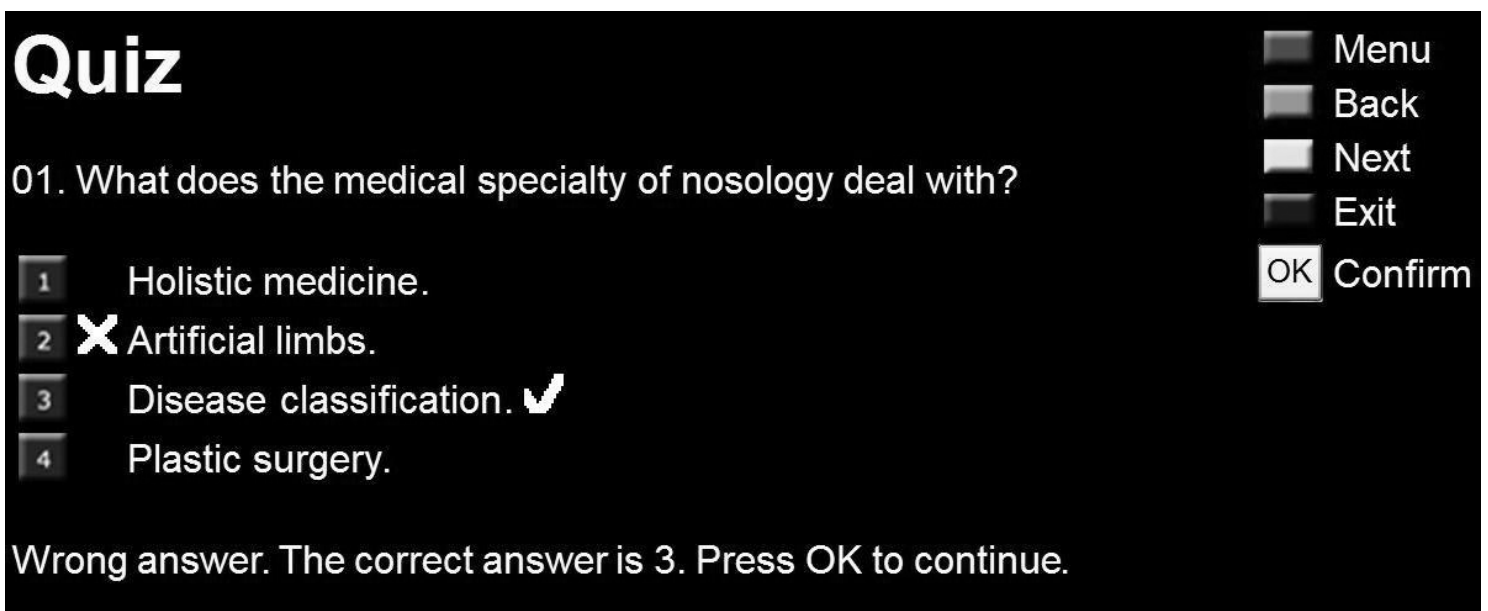

Figura 2. Tela do quiz 


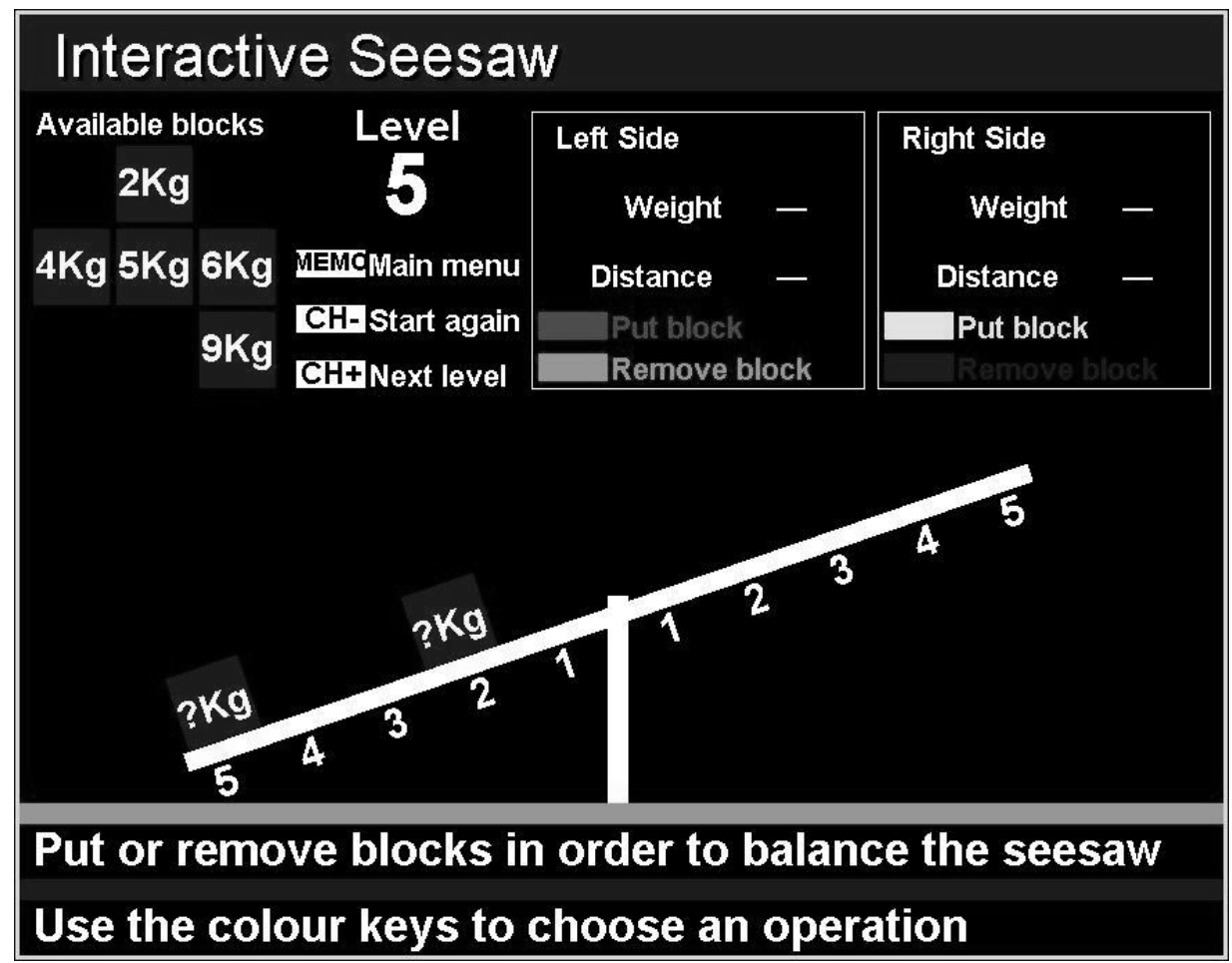

Figura 3. Tela da gangorra interativa.

Inicialmente, os protótipos passaram por uma avaliação heurística, que foi realizada por professores do Laboratório de Usabilidade da Universidade de Brighton, Reino Unido. Após a identificação e correção de problemas de usabilidade detectados na avaliação heurística, os testes de usabilidade foram realizados.

Os testes de usabilidade foram realizados no laboratório de usabilidade em TV digital interativa da citada universidade (ver figura 4). $\mathrm{O}$ equipamento do laboratório usado foi o mais discreto possível. As sessões foram gravadas através de duas câmeras CCTV e um pequeno microfone, sendo que a tela da TV também foi gravada.

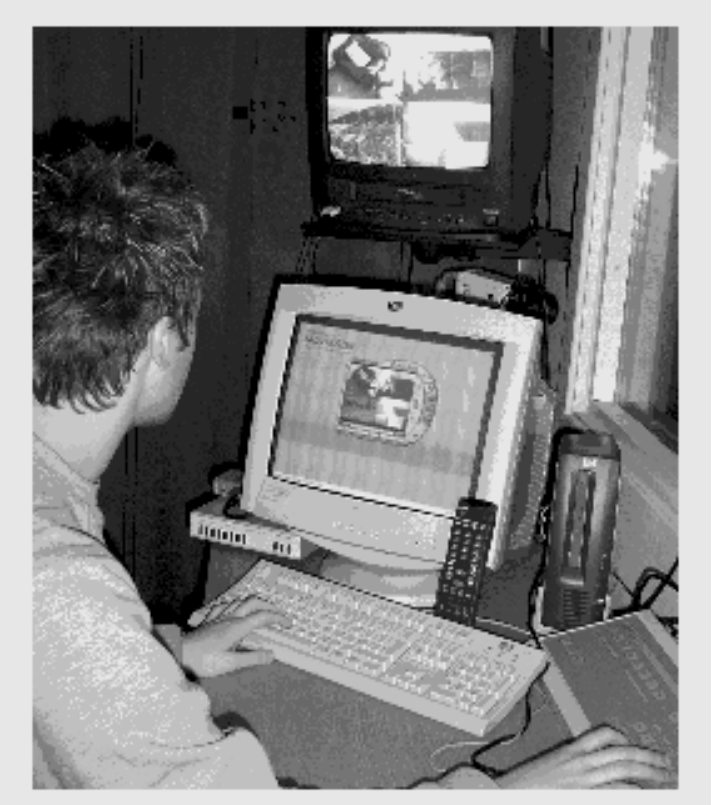

Figura 4: Equipamento do laboratório de usabilidade. 
Os participantes dos testes de usabilidade foram estudantes da universidade. Dos 8 participantes, 2 eram estudantes de graduação e 8 de pós-graduação, sendo 5 homens e 3 mulheres. Todos já tiveram experiência com TV digital, 5 deles já utilizaram objetos de aprendizagem no computador e apenas 3 já haviam utilizado objetos de aprendizagem na TV. Eram de nacionalidades diversas: Sudão (1), Iêmen (1), Arábia Saudita (1), País de Gales (1), Zimbábue (1), Grécia (2) e Inglaterra (1). A média de idade foi de aproximadamente 27 anos, sendo o mais jovem com 21 anos e o mais idoso com 46 anos.

O primeiro protótipo a ser colocado em testes foi a "gangorra interativa". O protótipo estava sendo executado em um computador pessoal, mas era mostrado em uma tela de TV. Para permitir a interação com o protótipo usando o controle remoto da $\mathrm{TV}$, os sinais do controle remoto foram mapeados para sinais de digitação no teclado do computador pessoal, usando o software PC Remote Control. Para a transferência do sinal do controle remoto, um receptor infravermelho foi conectado ao computador pessoal. Dessa forma, a interação com o protótipo foi muito próxima da realidade, pois, os participantes não notaram que estavam interagindo com uma aplicação executando em um computador pessoal, ao invés de uma aplicação executando em um set-top box.

Para os testes de usabilidade, os métodos de observação do usuário, thinkingaloud, análise de áudio e vídeo e questionário de usabilidade (Nielsen, 1993) foram aplicados. Durante o experimento, os estudantes estavam sozinhos, mas o processo de utilização dos objetos de aprendizagem foi gravado com a permissão do estudante. $\mathrm{O}$ objetivo da gravação é verificar possíveis problemas em uma parte particular do objeto de aprendizagem ou na navegação com o controle remoto.

A sessão de testes de usabilidade consistiu de três fases. Na primeira fase, os usuários exploraram livremente cada protótipo e obtiveram familiaridade com a navegação através do controle remoto. Na segunda fase, cada protótipo foi apresentado sem pedir ao usuário para realizar tarefas específicas. Seguindo o protocolo thinkingaloud, aos participantes foi pedido que verbalizem continuamente suas idéias, opiniões, expectativas, dúvidas, descobertas etc. durante o uso dos protótipos. Na terceira fase da sessão de testes, os usuários responderam um questionário de usabilidade (ver figura 5).
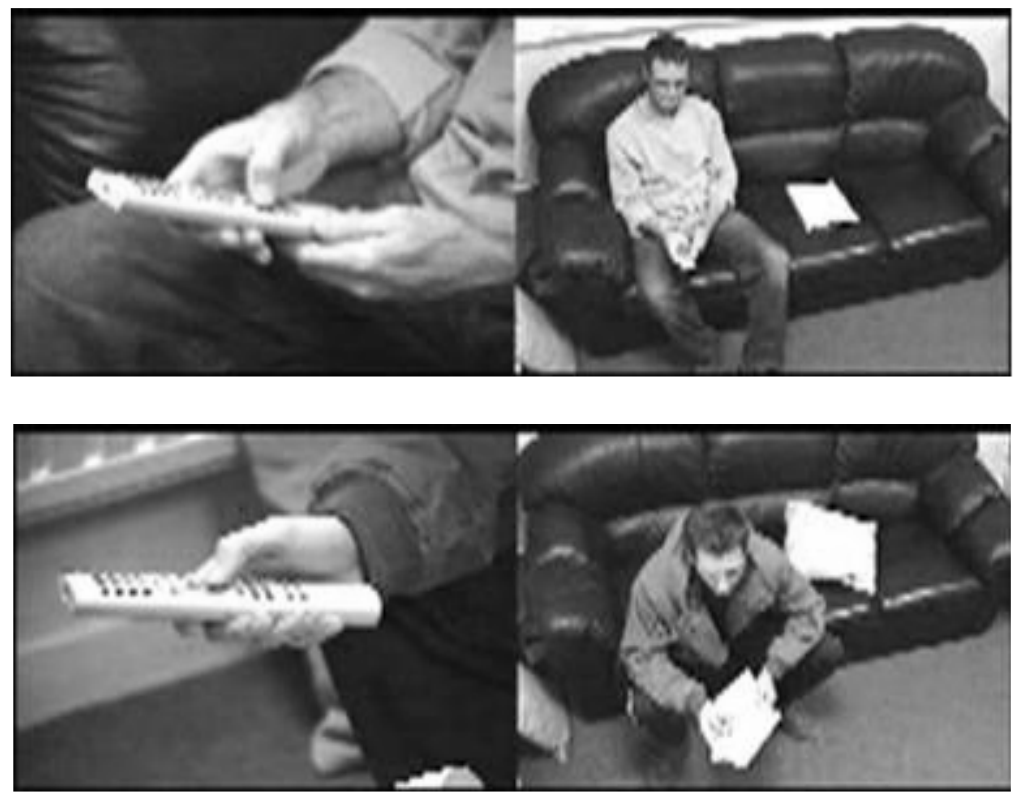

Figura 5. Usuários nas sessões dos testes de usabilidade. 
Os outros 2 protótipos serão colocados brevemente em testes. Também se pretende realizar testes de usabilidade similares com participantes brasileiros, com a finalidade de se comparar resultados e dar maior qualidade e precisão às orientações a serem propostas. $\mathrm{O}$ vídeo e os resultados dos testes de usabilidade serão posteriormente analisados para produzir as orientações de usabilidade para objetos de aprendizagem para TVD.

\section{CONCLUSÕES}

O objetivo da pesquisa apresentada neste artigo é suportar o desenvolvimento de objetos de aprendizagem fáceis de serem usados na TVD, fornecendo orientações de usabilidade para a implementação destes objetos.

Esta pesquisa irá contribuir para o campo do projeto centrado no usuário e seus resultados poderão influenciar nas interfaces dos objetos de aprendizagem que serão produzidos para TVD, como também, poderão ser úteis para redes de TV, indústria de software e designers em geral.

Pretende-se que se as orientações propostas forem aplicadas nas interfaces dos objetos de aprendizagem, estas poderão contribuir para o processo de ensino e aprendizagem, pois tornarão o objeto de aprendizagem mais fácil de ser usado. A facilidade de uso de uma aplicação de e-learning, por exemplo, de um objeto de aprendizagem, foi identificada como um dos motivos para a não-desistência do aluno em uma aplicação de e-learning em uso (Costabile et al, 2005).

\section{AGRADECIMENTOS}

Agradecimentos à Coordenação de Aperfeiçoamento de Pessoal de Ensino Superior (CAPES) pelo apoio financeiro através do Programa de Doutorado no País com Estágio no Exterior (PDEE).

\section{REFERÊNCIAS}

AARRENIEMI-JOKIPELTO, P. (2006). Modeling and Content Production of Distance Learning Concept for Interactive Digital Television. PhD thesis. Department of Computer Science and Engineering, Helsinki University of Technology, Finland.

AHOREN, A., TURKKI, L., SAARIJÄRVI, M., LAHTI, M., VIRTANEN, T. (2006). Guidelines for designing easy-to-use interactive television services: experiences from the ArviD programme, Proceedings of the 4th European Conference on Interactive Television, EuroITV 2006, Athens, Greece.

BAAS, K., VAN DEN EIJNDE, J., JUNGER, J., (2001). A practical model for the development of web based interactive courses, Proceedings of the 31st Annual Frontiers in Education Conference, Volume 1, 10-13 Oct. 2001.

BATES, P. J. (2003). A study into TV-based interactive learning to the home. pjb Associates, UK. This study has been conducted with funding from the European Community under the IST Programme (1998-2002).

BERGLUND, A; BERGLUND, E.; LARSSON, A.; BANG, M. (2005). The Paper Remote: An Augmented TV Guide and Remote Control. International Journal: Universal Access in the Information Society (UAIS), Springer-Verlag Heidelberg.

BBCi-Interactive Television Style Guide.(2002).Versão 2.1.

BRASIL (2006). Decreto n. 5.820, de 29 de junho de 2006. "Dispõe sobre a Implantação do Sistema Brasileiro de Televisão Digital - Terrestre, e dá outras providências". DOU, Brasília, 30 de junho 2006.

BRECHT, R., KUNERT, T. (2005) User requirements and design guidance for interactive TV news applications, Proceedings of the 3rd European Conference on Interactive Television, EuroITV 2005, Aalborg, Denmark. 
CARMICHAEL, A. (1999) Style guide for the design of interactive television services for elderly viewers, Disponível em <http://www.computing.dundee.ac.uk/projects/utopia/publications/Carmichael\%20\%20DesignStyleGuideFinal.pdf >, Acesso em 01 set 2007. CGIBR - COMITÊ GESTOR DE INTERNET NA BRASIL. (2006) Disponível em <http://www.cetic.br/usuarios/tic/2006/rel-geral-05.htm>. Acesso em 01 set 2007.

CHOI, H.; CHOI, M.; KIM, J.; YU, H. (2003). An empirical study on the adoption of information appliances with a focus on interactive TV. Telematics and Informatics, 20(2).

COSTABILE, M., DE MARSICO, M., LANZILOTTI, R., PLANTAMURA, V., ROSELLI, T. (2005) On the Usability Evaluation of E-Learning Applications, Proceedings of the 38th Annual Hawaii International Conference on System Sciences, 3-6 Jan. 2005.

DAMÁSIO, M. J. (2003). Uses of Interactive Television on Educational Settings: Evaluating the Media Impact. In Proceedings of the European Conference on Interactive Television, EuroITV2003, Brighton, United Kingdom.

FREEMAN, J.; LESSITER, J. (2003). Using attitude based segmentation to better understand viewer's usability issues with digital and interactive TV. In Proceedings of the 1st European Conference on Interactive Television, EuroITV, Brighton, United Kingdom.

IEEE LEARNING TECNOLOGY STANDARD COMMITTEE (LTSC). (2005) In: WG12 Learning Object Metadata. Disponível em <http://ltsc.ieee.org/wg12>. Acesso em 01 set 2007.

ISO 9241-11. (1998) Ergonomics requirements for office work with visual display terminals (VDTs) - Part 11: Guidance on usability. International Standard.

KUNERT, T.; KRÖMKER, H. (2007). Navigation Design Guidelines for Interactive Television Applications. Adjunct Proceedings of the 5th European Conference on Interactive Television, EuroITV 2007, Amsterdam, Netherlands.

LEE, B., LEE, S., (2002) Satellite remote education system design and implementation using MPEG-4 under DVB compliant network, Proceedings of 2002 IEEE International conference on Multimedia and Expo, 26-29 Aug. 2002.

LYTRAS, M.; LOUGOS, C.; CHIZOS, P.; POULOUDI, A. (2002) Interactive Television and e-Learning Convergence: Examining the Potential of t-Learning. European Conference on eLearning.

MENEZES, C. S.; LIRA, A. F.; FERRETTI, C.; LINDNER, E. L. (2006) ODAI - Objetos Digitais para Aprendizagem Interacionista. Anais do XVII Simpósio Brasileiro em Informática na Educação, 2006.

MINISTÉRIO DAS COMUNICAÇÕES. (2003) Política para adoção de tecnologia digital no serviço de televisão. Brasília, 2003.

NELSON, M. (1999). Remote controls. DigiPoints: The Digital Knowledge Handbook, 3(4).

NIELSEN, J. (1993) Usability Engineering. AP Professional, Cambridge, MA, SA.

NIELSEN, J. (1994). Heuristic evaluation. In Nielsen, J., and Mack, R.L. (Eds.), Usability Inspection Methods. John Wiley \& Sons, New York, NY.

PARKER, R. (1999) The Economics of Digital TV's future, In D. Gerbarg, The economics, technology and content of digital TV, 1999, USA: Kluwer Academic Publishers.

PERATON, H., CREED, C. (2000). Applying New Technologies and Cost-Effective Delivery Systems in Basic Education, World Education Forum, Education for All 2000 Assessment, Thematic Studies, UNESCO, 2000. Acesso em 01 set 2007. Disponível em <http://unesdoc.unesco.org/images/0012/001234/123482e.pdf>.

PIAGET, J. (1996). Biologia e conhecimento. 2a . edição. Petrópolis: Vozes, 1996.

RINNETMÄKI, M. (2004) A guide for digital TV service producers, 2004, ArviD publications 02/2004, Helsinki, Finland: Ministry of Transport and Communications.

RNIB (2005), Guidelines for the design of accessible information and communication technology systems, 2005, Royal National Institute for the Blind, Disponível em <http://www.tiresias.org/guidelines/television.htm>, Acesso em 01 set 2007.

WILEY, D. A (2001). Connecting learning objects to instructional design theory: A definition a metaphor, and a taxonomy. Acesso em 27 nov 2006. Disponível em: $<\mathrm{http}: / /$ reusability.org/read/chapters/wiley.doc $>$. 